\title{
¿POR Qué Bogotá No TIENE AL MENOS UNA LÍNEA METRO? Y LAS RAZONES POR LAS QUE PROBABLEMENTE NO LA TENDRÁ
}

Óscar A. Alfonso Roa*

\section{Resumen}

No obstante que la decisión sobre la primera línea metro para Bogotá se esbozó hace 75 años, en la actualidad la ciudad no cuenta con ella, pero tampoco existe una reflexión académica acerca de las razones para tal rezago. Durante este lapso de tiempo, el capital físico e institucional destinado a la movilidad se ha deteriorado al punto de llevar al colapso al sistema de transporte masivo de pasajeros, que tiene varias manifestaciones, siendo las más notables el incremento sustancial en los tiempos improductivos de viaje, la congestión vehicular, la saturación del parque automotor destinado a la movilidad colectiva y la inseguridad personal a la que se exponen los usuarios. En este artículo se sustenta la idea de que en una metrópoli de ocho millones de habitantes, con poderosas inflexibilidades infraestructurales, el trazado de la línea prioritaria del metro debe resolverse en favor de conectar las zonas más densamente pobladas de la ciudad, con lo que se resolverían secuencialmente los rezagos de inversión y se dotaría de mayor sostenibilidad ecológica y financiera al sistema de transporte masivo de pasajeros.

Palabras clave: movilidad cotidiana, sistema metro, política de transporte masivo de pasajeros, teoría de la inversión pública, Bogotá.

* Doctor en Planeamiento Urbano y Regional, Economista. Profesor Ordinario - Investigador. Grupo Construcción de Estado, Territorio y Paz, Universidad Externado de Colombia. Bogotá (Colombia). [oscar.alfonso@ uexternado.edu.co]

Recibido: 24 de junio de 2016 / Modificado: 30 de agosto de 2016 / Aceptado: 1 de septiembre de 2016

Para citar este artículo

Alfonso Roa, O. A. (2016). ¿Por qué Bogotá no tiene al menos una línea metro? Y las razones por las que probablemente no la tendrá. OPERA, 19, pp. 139-159.

DOI: http://dx.doi.org/10.18601/16578651.n19.08 


\section{WHY DOES BOGOTA NOT HAVE AT LEAST ONE METRO LINE? \\ AND THE REASONS WHY IT WILL PROBABLY NOT HAVE ONE}

\section{Abstract}

Although the decision on the first metro line in Bogotá was first sketched out 75 years ago, the city still does not have it, but there is no academic reflection on the reasons for such delay. During this period, the physical and institutional capital dedicated to mobility has deteriorated to the point of capsizing the system of mass passenger transport. The result is visible in the substantial increase in unproductive travel time, traffic congestion, saturation of the fleet intended for collective mobility and personal insecurity to which users are exposed. This article supports the idea that in a metropolis of eight million people, with powerful infrastructure inflexibility, the layout of the priority metro line should be resolved in favor of connecting the most densely populated areas of the city, which would progressively solve investment lags and would provide increased ecological and financial sustainability of the system of mass transit.

Key words: Daily mobility, metro system, policy of mass passenger transport, public investment theory, Bogota.

El colapso de la movilidad cotidiana de pasajeros en Bogotá es evidente: el incremento sustancial en los tiempos improductivos de viaje que, según el Departamento Nacional de Planeación (DNP) y Global Green Growth International, asciende a siete millones de horas al año, y la congestión vehicular que según las mismas fuentes cobra el $2 \%$ del PIB en Colombia, son algunas de las manifestaciones más relevantes. En otra, como el incremento en la motorización privada, hay notables desacuerdos estadísticos oficiales, mas no por ello deja de tener trascendencia: según la Secretaría Distrital de Movilidad (2014), circulan un millón de automóviles particulares y 418 mil motocicletas, mientras que para DNP-GGGI hay 1,4 millones de particulares y casi dos millones de motocicletas. Decisiones como la de la flexibilización del contrato de trabajo incrementaron considerablemente la demanda de movilidad cotidiana (Alfonso, 2015b). En este contexto, el inicio de las obras de la primera línea del metro de Bogotá aparece para muchos como la solución a los déficits acumulados de capital para la movilidad. En el presente análisis se sostiene que la línea prioritaria del metro es una solución eficaz para los problemas que enfrentan miles de pasajeros que residen en las zonas más densas de la ciudad, siempre y cuando el trazado las involucre, pero que no resuelve otros problemas como el de la congestión.

Para explicar por qué Bogotá no cuenta con al menos una línea metro, se recurre a una teoría de la inversión que explique los rezagos de decisión, de financiación y de instalación distribuidos en el tiempo, a fin de analizar las indefiniciones y los riesgos no asumidos desde el modelo público comercial y que, además, sugiere unas razones por las que probablemente no la tendrá. La historia de la inexistente primera línea del metro 
para Bogotá se presenta en la primera parte, mientras que en la segunda se exploran los vínculos teóricos entre la gestión comercial del transporte masivo de pasajeros y la teoría de la inversión productiva aludida para, en la tercera, presentar un balance de los rezagos en algunas experiencias del continente americano. En las reflexiones finales se sugiere una línea de acción política para enfrentar el rezago de decisión y, de allí, también los de financiación e instalación.

\section{UNA HISTORIA DE LA INEXISTENTE PRIMERA LÍNEA METRO PARA BOGOTÁ}

La demanda de movilidad cotidiana ha crecido sustancialmente desde que se tuvo la idea original de una línea metro para Bogotá hace 75 años y el tamaño de la ciudad era alrededor del $10 \%$ de lo que es en la actualidad, tanto en su área urbana como en su población. La demanda de movilidad se ha ampliado considerablemente: en la última década, por ejemplo, el número de viajes se ha ampliado a una tasa promedio anual del 7,5\% (Secretaría de Distrital de Movilidad, 2014, p. 137). Adicionalmente, las densidades de población se fueron apartando del sendero óptimo sugerido por los teóricos de la ciudad monocéntrica, caracterizada por una silueta elevada de edificaciones en el distrito comercial central que decae a medida que se incrementa la distancia hacia las zonas periféricas. No hay un ideal de densidades, pero su adecuada gestión es un comienzo de la garantía de movilidad que es una de las preocupaciones centrales en las metrópolis del mundo (Clark y Moir, 2015), garantía comúnmente entendida como la "movilidad sostenible" (Duarte, 2015). Los sistemas metro son eficaces en metrópolis con zonas muy densas (Mohan, 2008).

La prolongación del rezago de decisión sobre la primera línea metro ha dejado secuelas difícilmente superables. Una decisión sobre el trazado, la tecnología, el número de estaciones y el área de aferencia a las estaciones era más simple cuando la ciudad tenía alrededor de 380.000 habitantes como ocurría en 1942, y otra bien diferente es la que se piensa en la actualidad cuando la ciudad se apresta a acoger ocho millones y, en algo menos de diez años, tendrá un millón adicional. Las decisiones se complejizan a medida que las más decisivas se aplazan de manera reiterada y, por ello, hay cada vez más opiniones sobre cuál sería la mejor opción para una metrópoli que se densifica pero que también se expande hacia su entorno inmediato, la Sabana de Bogotá. La oleada de opiniones, muchas de ellas emitidas al calor de los intereses creados, tiene la potencia de agrandar el maremagnum: se desconoce lo que se necesita.

Una metrópoli de ocho millones de habitantes no tiene que tener necesariamente el mismo trazado de otra semejante; es decir, el de Lima no tiene por qué ser semejante al de Bogotá. Menos con el de Medellín, que es empleado en los debates públicos como referente en cuanto a su trazado y expansión. ¿Por qué se recurre a esta comparación? El tamaño de la población de Medellín es 3,17 veces inferior al de Bogotá y goza de una geografía urbana sustancialmente diferente: el Valle de Aburrá está fragmentado en 12 jurisdicciones cuyas cabeceras son tan contiguas que hasta 
la Maratón de Medellín atraviesa por tres de ellas -Envigado, Itagüí y Sabaneta-, algo mucho más difícil en la Sabana pues sus cabeceras son dispersas. El área de las cabeceras municipales de las jurisdicciones del Valle de Aburrá es de $204,3 \mathrm{~km}^{2}$, que es el 11,6\% de su área total, mientras que en las de Bogotá y la Sabana suman $498,3 \mathrm{~km}^{2}$ y son el $10,4 \%$ de su área total. El área total de Bogotá y la Sabana es 2,7 veces superior a la de Medellín y el Valle de Aburrá. Es por ello que, en caso de que el metro de Bogotá alcance contornos metropolitanos, tendrá necesariamente que encarar rutas con prolongados tramos sin estaciones. La salida es evidente y no se necesitan costosos estudios que la releven: en la Sabana se requiere de un tren de cercanías que se integre en algún lugar con el metro de Bogotá, cuando exista.

Abundan los balances y las validaciones sobre las iniciativas para la primera línea metro (Secretaría Distrital de Planeación, 2008; Universidad Nacional de Colombia, 2010; Suárez, 2015). Los estudios de Sofretu-Ineco-CS (1981), InterMetro SpA (1987), JICA (1996), Systra-Bechtel-Ingetec (1997), Sener-TMB-Advancede Logistic Group-Incoplan-Santander Investment Colombia y Garrigues Abogados (2009) y Euroestudios-Idom Ingeniería-Cano Jiménez (2013) no concuerdan en casi nada. A manera de ejemplo, el realizado en 1997 intentó asemejarse sin éxito al de 1981 y no a su antecedente más cercano, el elaborado por JICA en 1996. El intento de advertir el futuro de la ciudad con el metro ha sido uno de los principales traspiés de las iniciativas. La ausencia de una orientación del desarrollo de la ciudad, la incoherencia de las propuestas con los ulteriores trazados de los planes de ordenamiento territorial, o el uso del sistema para la producción de suelo urbano con miras a la renovación urbana son algunos de los vacíos que han advertido tales balances y validaciones. En la actualidad, Systra ha sido contratada para "precisar las alternativas de trazados y longitudes para el tramo oriental" (Escobar, 2016). Esto ya había sido hecho hace veinte años por la misma empresa y las firmas consorciadas, pero la novedad es que en la actualidad la ciudad se ha modificado, tiene la incertidumbre del contenido del nuevo РОт y un sistema integrado de transporte rodeado de dilemas. La historia se repite. Nuevas recomendaciones y más desaciertos, y el rezago de decisión continúa latente. El actual alcalde anunció que la primera línea metro llegaría hasta Mosquera en el occidente, pero el estudio contratado con Systra se refiere al tramo oriental.

Otros elementos del sistema tales como el número de estaciones, los tramos superficiales y los subterráneos, además del trazado y la extensión, no gozan de unanimidad en ninguno de los estudios. Se desconocen aspectos cruciales como las áreas de aferencia de pasajeros y, por tanto, la velocidad comercial que puedan desarrollar los trenes. Las encuestas de origen-destino que han servido de soporte a los últimos estudios tienen deficiencias estadísticas originadas tanto en el muestreo como en la aplicación de los instrumentos de recolección. Los microdatos de la Encuesta Origen-Destino no se encuentran disponibles, pues la actual administración está ajustando los que obtuvo la pasada administración. La estadística sobre movilidad cotidiana se 
politizó y pierde credibilidad, pues tanto los ascensos y descensos como los orígenes y destinos de pasajeros son registros que no requieren imputaciones de ninguna índole (Mauttone, 2005, pp. 108 y ss.).

No obstante el rezago de decisión, en Bogotá se adelanta el proceso de financiación de la primera línea metro, pasándose por alto algunos dogmas financieros como aquel que predice que cualquier cambio de opinión hará que se incrementen los costos del proyecto. Y muchos cambios harán sus finanzas insostenibles. Mediante el Decreto 1008 de 2005, el Gobierno nacional reglamentó el Servicio de Transporte Masivo de Pasajeros en el que se define explícitamente la modalidad del metro ligero y, de manera subsidiaria, el metro pesado. En ningún caso se contempla el "metro elevado" con el que se empecinó el actual alcalde, so pretexto de que este calificativo hace referencia a los "tramos" superficiales del sistema, por oposición a los subterráneos. Este no es un pseudoproblema semántico debido a que el numeral segundo del artículo segundo de la Ley 310 de 1996, establece una condición ineludible para que la nación cofinancie o participe en el sistema: que esté clara la factibilidad técnico-económica, socioambiental y físico-espacial, así como la rentabilidad y la estrategia del Sistema Integrado de Transporte Masivo propuesto. Las evaluaciones internacionales explican las razones por las que las inversiones en un metro convencional son cuatro veces superiores a las que requiere un metro ligero (Alonso-Neira et al., 2013).

Precisamente, esa norma estableció que la nación participaría mínimo con el 40 \% y máximo con el $70 \%$ del servicio de la deuda del proyecto, al cumplirse esas condiciones y otras como la constitución de una sociedad por acciones que sea titular del sistema, sujetando la garantía de los créditos externos a la pignoración de las rentas locales con las que se cubra el remanente a cargo de la ciudad, pudiéndose cubrir con incrementos tarifarios, peajes urbanos, gravámenes a los parqueaderos $\mathrm{y}$, finalmente, abre las posibilidades de dar en concesión tramos del sistema. Quince ańos atrás, el DNP (Conpes, 1982, p. 19) recomendó que el Proyecto Metro para Bogotá fuera financiado totalmente con los recursos de la ciudad. En abril de 2016, el gerente del metro previó su entrada en operación en 2020 (Escobar, 2016), pero en septiembre el presidente de la república y el alcalde mayor acordaron que sería en 2022.

En cuanto a la factibilidad económica, el Conpes sugirió en 1982 que el proyecto metro alcanzaría una tasa interna de retorno (TIR) del 14,2\%, mientras que en 1998 la estimó en $15,8 \%$, "incluyendo en el flujo los costos de operación estimados para la PLM y para el sistema de buses integrado, así como los costos de reposición" (Conpes, 1998, p. 16); ya en el 2000 (Conpes, 2000, p. 6), la TIR se estimó en $11,3 \%$. Por su parte, la administración Petro estimó en 6,02\% la tasa de descuento del proyecto incluyendo el "riesgo país" (Alcaldía Mayor de Bogotá - Metro de Bogotá, s. f., p. 324), límite inferior de una tasa de retorno social que no se conoce.

Otro tipo de riesgos afloran como resultado del rezago de la decisión. El Conpes (1998, p. 20) incorporó el de adquisición de predios, cuya adecuada gestión exige de 
una planeación proactiva con la que la ciudad goce del tiempo suficiente para realizar unas negociaciones que, de no culminar por consenso entre las partes, terminan en prolongados procesos de expropiación por vía administrativa durante los cuales las obras se suspenden, al menos en los tramos en donde los litigios no hayan concluido. Los riesgos de obra civil fueron reconocidos por el Conpes en 1982 y 1998. Otros riesgos conexos que se han considerado eventualmente son los derivados de los incrementos en el precio de los combustibles y de la mano de obra, así como los costos incurridos en la obtención de la licencia ambiental.

Los riesgos comerciales en los proyectos de infraestructura ocurren por la subestimación de los costos y la sobreestimación de los beneficios (Flyvberg, Garbuio y Lovallo, 2009). En el caso del metro están asociados a las previsiones acerca del volumen de pasajeros movilizados. En 1982 se preveían 1,25 millones de pasajeros / día (Conpes, 1982, p. 11), mientras que en 1998 (Conpes, 1998, p. 12) se estimó que alcanzaría 353 mil pasajeros / día en 2001, hasta llegar a 1,13 en 2008 cuando se saturaría la línea prioritaria. En el balance ex ante de la administración Petro (Alcaldía Mayor de Bogotá - Metro de Bogotá, s. f., p. 421), se estimó que el número de abordajes iniciales sin transferencia sería de 376.900, más un porcentaje incremental anual de la amplitud del rango (690.240 - 376.900), estimándose que en el 2050 los abordajes con transferencia llegarían a 743.580. Las diferencias son considerables debido a que se trata de trazados diferentes. Si algo se puede sacar en claro, es que los trazados por las zonas más densas son los que proveerían el mayor número de abordajes y con ello, se cumpliría el objetivo crucial enunciado, cual es el de solucionar el problema de movilidad cotidiana al mayor número posible de usuarios. Realizar un trazado por zonas poco densas y con escaso potencial para densificarse introduce un riesgo de demanda que, por diferentes razones, ha tratado de justificarse con ciertas interpretaciones de los resultados de las encuestas de origen-destino.

El sistema metro es socialmente más deseable pues alcanza un ratio positivo en número de pasajeros transportados y, dependiendo del trazado y la tecnología, en la velocidad comercial, frente al vehículo particular. Sin embargo, hay un imponderable que no puede ser subvalorado: que el sistema metro no mejore la congestión o que, en el peor de los casos, la agrave. Thomson (1997, p. 108) advirtió sobre este fenómeno. Aquellos usuarios del vehículo privado que cambian al modo metro, liberan espacios en las vías y en los lugares de parqueo que alientan a otros usuarios potenciales del vehículo particular a usarlos; es decir, el anuncio de la descongestión vehicular alienta a usar el vehículo privado. Esta conducta puede ser igualmente estimulada por la reducción del parque automotor de buses resultante del traslado de una porción significativa de pasajeros al sistema más moderno, que contribuye a la liberación de espacio vial para nuevos vehículos privados. En las áreas de influencia inmediata a las estaciones del metro, los cambios de uso del suelo a actividades más rentables, predominantemente comerciales, induce a que propietarios y trabajadores copen amplias 
zonas otrora residenciales, por ejemplo, con lo que la afluencia de vehículos particulares se incrementa, y lo hace aún más si, como es de esperarse, la densificación residencial en altura acompańa esos cambios de uso del suelo con precios más elevados y, con ello, la probabilidad de que la congestión vehicular se incremente en tales zonas.

El incremento en la demanda de este tipo de transporte colectivo y la reducción en el uso del automóvil privado no son inmediatos (Weisbrod, 2009) y requieren de gestión y regulación urbanas. La regulación urbanística es más eficaz utilizando el "máximo de parqueos" y no algún mínimo como usualmente ocurre (Thomson, 1997, p. 110). El sistema metro aumenta paulatinamente la demanda de movilidad -en $15 \%$ (De Grange, 2010) - y por ello sobreviene la densificación de otras zonas a lo largo de su trazado. No obstante, existen rigideces e inflexibilidades de ciertos segmentos de la población a emplear el sistema metro que obedecen a diferentes razones, como la preferencia por el automóvil particular por las personas de edad avanzada que residen en esos vecindarios, como también por la creencia de que sobrevendría cierto deterioro social con la instalación de las estaciones; sin embargo, una que es pocas veces analizada es la dotación de parqueos privados por vivienda que, de hecho, se ha erigido como un elemento que discrimina poderosamente según la capacidad de gasto de los hogares y el segmento residencial al que pertenecen (Alfonso, 2015a; Alfonso y Amézquita, 2016). Esa regulación impactaría positivamente la congestión vehicular y la demanda por el sistema de transporte colectivo.
En los segmentos más elevados de Bogotá, los de los hogares con mayor capacidad de gasto, se han producido viviendas hasta con diez lugares de parqueo privado, mientras que en los segmentos con capacidad de gasto baja o virtualmente inexistente, esa relación es de un parqueo por cada cinco y hasta diez viviendas. La renta de los parqueos es del mismo nivel, como mínimo, que la renta de segregación de la vivienda (Jaramillo, 2009) y, por tanto, los miembros del hogar pudiente difícilmente renunciarán a usufructuar ese espacio.

Esta breve descripción de las razones por las cuales Bogotá no cuenta aún con una línea metro será analizada a continuación, a partir de una teoría que explica los rezagos en la inversión productiva que, para el efecto, es decidida desde un ámbito de la gestión comercial de los servicios públicos, en el entendido de que este es el modelo que orienta las decisiones del sistema.

\section{EL MODELO PÚBLICO COMERCIAL DE LA PRESTACIÓN DE SERVICIOS PÚBLICOS Y COLECTIVOS POR EL ESTADO Y LA TEORÍA DE LA INVERSIÓN}

El modelo público comercial es una de las formas dominantes que en la actualidad adopta la prestación de algún servicio público o colectivo y que, a diferencia de otras prácticas monopólicas del Estado asociadas generalmente a la explotación de alguna rama de la economía, tales como la producción minera y de hidrocarburos, su ciclo de vida tiene un horizonte temporal muy prolongado. 


\section{La inversión en un modelo público comercial de prestación de los servicios públicos y colectivos}

Los enclaves productivos se agotan cuando el subsuelo ya no es depositario de alguna riqueza o cuando precios muy bajos no permiten su explotación. Por su parte, la competencia puede arruinar a algún monopolio estatal, como el de los licores, por razones de eficiencia y, además, por la injerencia de la política en las decisiones comerciales. La prestación domiciliaria de los servicios de acueducto y saneamiento básico -alcantarillado pluvial y sanitario, y recolección y disposición final de basuras-, así como la energía eléctrica y la telefonía fija, adoptan ese esquema sin que la responsabilidad del Estado desaparezca por tal motivo. Desde 1994, con la promulgación de la Ley 142, ese modelo se ha afianzado, quedando en claro que la prestación directa por el Estado -los entes territoriales, municipios o departamentos- son la última de las opciones por considerar. Esto ocurre por dos razones: primero, la desconfianza de quienes ejercen el nivel central de Gobierno en los entes territoriales acerca de su capacidad empresarial, traducida generalmente como "eficiencia en la prestación", y, segundo, por la existencia de capitales sobreacumulados en todo el planeta que demandan un espacio económico para valorizarse. A lo anterior podría sumársele esa onda ideológica tan arraigada entre la tecnocracia y propalada por ella acerca de que la privatización es el mejor de los mundos posibles.

Ese modelo también se replica en la movilización colectiva de pasajeros, y la es- tructuración, puesta en marcha y operación del sistema metro no puede ser la excepción. Dos aspectos requieren precisarse. El primero es que no por ser público se realiza a fondo perdido; por el contrario, la remuneración al capital es indeclinable por más que este servicio esté dentro de la órbita social de la acción estatal. La regla financiera al respecto es simple: la remuneración al capital debe ser superior al costo financiero de los capitales tomados en préstamo pues, de lo contrario, el proyecto asumirá pérdidas financieras que en una situación extrema pueden llevar al colapso financiero del proyecto. ¿Cómo se toma esta decisión? Las bancas de inversión proveen servicios a las entidades estatales cuyos calificados informes son revestidos de las más complejas técnicas y argumentos financieros. No obstante, como es una decisión que influye de manera decisiva en la fijación de la tarifa técnica la que, por su parte, permite formar la tarifa al público, trasciende esa esfera "técnica” para definirse en el ámbito político. Esta discusión se retomará cuando se discuta el rezago de financiación. El segundo deviene del anterior: como se persigue cierta remuneración al capital invertido, las reglas para la concepción del proyecto, su financiación, su instalación y su puesta en marcha, son las mismas que para cualquier proyecto privado de inversión productiva, pero ¿entienden esto los políticos? El modelo público comercial no está exento de la interferencia de la política $y$, en muchos casos, se somete a las reglas de la política porque en esa esfera se cuenta con la capacidad de movilizar un poderoso instrumento -el presupuesto- para sufragar los sobrecostos que se derivan de sus decisiones. 
La política jamás ahorra un décimo, siempre incrementa el costo de las decisiones.

El modelo público comercial para el sistema metro, tal como lo establece el primer numeral del artículo segundo de la Ley 310 de 1996, opera bajo la forma jurídica de una sociedad por acciones. La Empresa Metro de Bogotá S. A. fue autorizada por el Concejo de Bogotá y deberá estar constituida y operando antes del 26 de abril del 2017, y sobre ella recaerá "la planeación, estructuración, construcción, operación, explotación y mantenimiento de las líneas de metro que hacen parte del Sistema Integrado de Transporte Público de Bogotá, cumpliendo con las normas ambientales, urbanísticas, arquitectónicas, de sismorresistencia y técnicas. Así como la adquisición, operación, explotación, mantenimiento, y administración de los trenes" ${ }^{1}$. Simultáneamente, se asignaron $\$ 4,1$ billones de pesos (us 1.379 millones) con cargo a vigencias futuras a fin de que la ciudad cumpla con los requisitos de cofinanciación que exige la nación. Esta empresa quedó facultada para ejecutar proyectos de renovación urbana en el área de influencia del sistema, y para decretar el cobro de plusvalías urbanas derivadas del mayor precio del suelo atribuible a las obras, propósitos que se insertan dentro de los "fines de crecimiento y competitividad" que persiguen los grandes proyectos urbanos (Rodríguez y Abramo, 2011, p. 2). Debido a la magnitud de la inversión y a sus riesgos, los grandes proyectos urbanos son promovidos y gestionados por este tipo de modelos (Cuervo,
1994, p. 303), sin que ello signifique un desplazamiento de la inversión privada como lo advierten los enfoques dominantes de la teoría de la inversión (Oliveira y Texeira, 1999).

\section{Una teoría de la inversión}

El modelo público comercial se rige por las reglas del mercado, y por perseguir la eficiencia en la asignación de recursos. Para explicar los niveles de inversión, Bour (2004) sigue las reglas del principio de aceleración y retoma la idea de la relación inversa entre el costo de capital y la inversión, siempre en el marco del equilibrio macroeconómico en el que la oferta de fondos prestables es determinado por el ahorro. Pero Miller (1967) había advertido que dicho nivel depende de la continuidad de la demanda, de manera que si esa función es discontinua, la tasa de cambio de los activos de capital también lo es. En el caso de la primera línea metro (PLM), la agudeza de esa discontinuidad está estrechamente ligada al trazado con el que se pretenda atender la demanda insatisfecha de movilidad cotidiana.

La relación capital / producto $(v=K / Y)$ del sistema de movilidad de una ciudad monocéntrica es superior a la requerida en una ciudad policéntrica debido a que el policentrismo es característico de las grandes aglomeraciones que, por su parte, demanda grandes extensiones de suelo. Las variaciones porcentuales interurbanas del capital requerido para habilitar el sistema metro son menos que proporcionales a los incrementos

1 Véase http://app.idu.gov.co/seccion_metro_ASP/ 
en el flujo de caja originados en el mayor volumen de pasajeros que lo demandan en el segundo tipo de ciudades: los viajes anuales por pasajero se incrementan mientras que el promedio vagón / kilómetro recorrido disminuye (Alfonso, 2012b, p. 38). Cualquier incremento sustancial en la demanda $\left(Y_{t}-Y_{t-1}\right)$ por un bien o servicio origina un incremento en los precios en cualquier plazo, a no ser que se incremente su oferta para lo que se requiere incrementar el stock de capital $\left(K_{t}-K_{t-1}\right)$ en una proporción $(v)$ que, por regla general, es menor que el incremento en la demanda; es decir, que $v$ asume valores inferiores a la unidad. Ese incremento en el stock de capital es la inversión bruta (I).

$$
K_{t}-K_{t-1}=v\left(Y_{t}-Y_{t-1}\right)
$$

Desde esta perspectiva, los problemas de movilidad ocurren porque $v$ ha sido más próxima a cero que a la unidad. Los incrementos en la demanda de movilidad cotidiana han sido atendidos con variaciones marginales en el stock de capital que ocasionaron un incremento sustancial en el "costo pleno de transporte" (Wingo, 1972), esto es, en la tarifa pagada por el usuario adicionada con la elevación del costo de oportunidad del tiempo capturado por la ineficiencia del sistema de transporte colectivo. El stock de capital $K_{t}$ es inferior al requerido para prestar el servicio en óptimas condiciones $K_{t}^{*}$, por tanto, el rezago en la inversión se ha incrementado con el paso del tiempo. Siguiendo la notación de Ebour (2004):

$$
I_{t}=K_{t}-K_{t-1} ; I_{t}^{*}=K_{t}^{*}-K_{t}
$$

$$
K_{t}=\mu K_{t}^{*}-(1-\mu) K_{t-1}
$$

Puesto que $I_{t}^{*}=v\left(Y_{t}-Y_{t-1}\right)$, tal que $K_{t}^{*}-K_{t-1}=v\left(Y_{t}-Y_{t-1}\right)$, y dado que $K_{t-1}=$ $v Y_{t-1}$, se obtiene que:

$$
K_{t}^{*}=v Y_{t}
$$

Reemplazando en la ecuación (3) se obtiene que:

$$
K_{t}=\mu v Y_{t}+(1-\mu) K_{t-1}
$$

Los stocks de capital se reemplazan en forma iterada en la última ecuación para obtener la expresión del stock de capital requerido en el periodo $t$ :

$$
K_{t}=\mu v \sum_{i=1}^{\alpha}(1-\mu)^{i-1} Y_{t-1}
$$

De donde la inversión requerida en $t$ es:

$$
I_{t}=\mu v \sum_{i=1}^{\alpha}(1-\mu)^{i-1}\left(Y_{t-i}-Y_{t-i-1}\right)
$$

Esta última es una función de inversión que explica que ella es una fracción $(v)$ de los cambios ocurridos en la demanda de movilidad en el pasado, y que la inversión realizada es solamente una fracción de la inversión requerida $(\mu)$. Esta expresión de “rezagos distribuidos" que se acotan geométricamente sugiere que las discrepancias entre el stock de capital requerido y el actual se aproximan secuencialmente. Pero la celeridad del ajuste no ha sido la regla del modelo público comercial de gestión del transporte colectivo, de manera que los cambios en la demanda se han ido acumulando a tal punto que la brecha 
entre el stock de capital requerido y el actual es muy amplia.

Los economistas han realizado diversos aportes a la teoría de los rezagos distribuidos de la inversión productiva. Koyck (1954) fijó su interés en la comprensión de la velocidad a la que las necesidades del crecimiento del producto serán satisfechas con el incremento en la capacidad productiva (en Guitton, 1955, p. 128). Las velocidades de reacción confieren mayor o menor amplitud a los rezagos. Jorgenson (1967, p. 132) realiza un estado del arte del que se deduce la convergencia de hallazgos de los rezagos sobre el incremento en el precio de los bienes de inversión y las modificaciones en la tasa de descuento. Eisner y Nadiri (1968) realizaron pruebas econométricas para poner en duda la incidencia de los precios relativos como determinantes de las variaciones en la demanda de los bienes de inversión argumentada por Jorgenson, modelándose en todo caso con funciones Cobb-Douglas que suponen rendimientos decrecientes a escala. Tobin (1969) denominó $q$ al cociente resultante del valor de mercado de un activo y el de su reposición a nuevo, que sirve para orientar las decisiones empresariales sobre la tasa del ajuste de la inversión cuando se conoce el valor presente de los beneficios futuros de la firma.

El inicio del rezago debe establecerse desde una fecha cierta que es cuando se tiene certeza de que ocurrirá la variación en la demanda que requiere el ajuste del acervo de capital productivo a través de la inversión. Como el ajuste no es automático, aparecen los rezagos cuya amplitud depende de la celeridad con la que se tome la decisión de realizar tal ajuste, de la disponibilidad de recursos propios y del acceso al mercado de fondos prestables para financiarlo y, por último, de la gestión económica e ingenieril que facilite la puesta en operación de la nueva capacidad de planta. Estos tres rezagos - de decisión, de financiación y de instalación- serán analizados a continuación.

\section{LOS REZAGOS EN LA INVERSIÓN PRODUCTIVA SISTEMA METRO A LA LUZ DE ALGUNAS EXPERIENCIAS DE SISTEMAS METRO EN EL CONTINENTE AMERICANO}

La inversión requerida $(\mu)$ jamás será la óptima según la teoría de los rezagos distribuidos de la inversión, pero ella debe aproximarse a la necesaria según los incrementos ocurridos y previsibles en la demanda de movilidad $(v)$. Uno de los problemas más acuciantes que enfrenta la inmensa mayoría de los residentes en Bogotá y en los municipios de su área de influencia inmediata -la zona metropolitanaes la movilidad, entendida como el excesivo tiempo que demanda el acceso a los lugares cotidianos de desplazamiento, sea cual fuere el medio empleado. La incertidumbre sobre las frecuencias de circulación de los vehículos de transporte colectivo, la tarifa, la incultura ciudadana en el sistema integrado de transporte y la complejidad de los códigos para movilizarse en la ciudad, son fenómenos que el ciudadano generalmente atribuye a la movilidad. Otros, como la creciente accidentalidad y la inseguridad en los sistemas de transporte colectivo, son atribuidos también a la movilidad, aunque 
son del campo de las normas de tránsito y de los códigos de policía. Se entiende que el sistema metro contribuye a resolver el problema original y que los males conexos tendrán que resolverse simultáneamente pero no por causa exclusivamente de su entrada en operación.

El sistema metro es un componente del Sistema Integrado de Transporte Público (SITP), y no un sustituto. Es por esto que la solución a problemas como el de la congestión recae sobre el conjunto del Sistema, y no meramente sobre una de sus partes. La demanda de movilidad no es adecuadamente atendida por múltiples razones, como el estímulo en los contratos con los operadores del Transmilenio (TM) a ganar la tarifa por pasajero transportado y no por la frecuencia de despacho de los articulados y de los alimentadores, el inadecuado trazado de rutas o el uso desmedido del vehículo privado.

\section{Rezago de decisión}

Los sistemas metro han pasado por varias etapas tecnológicas. El antecedente más mencionado es el tranvía halado por equinos que luego fueron reemplazados por el impulso eléctrico, luego los monorrieles subterráneos y, en fin, hasta la actualidad en donde los campos electromagnéticos que evitan la fricción ha permitido un mejor desempeño aerodinámico de los trenes. En cada momento, esas innovaciones fueron una novedad y se incorporaron de manera versátil a los sistemas.

La idea original de un sistema metro para Buenos Aires data de 1886, habiéndose iniciado las obras en 1909 hasta que en 1913, cuando la ciudad contaba con 1,6 millones de habitantes, fue inaugurado el primer trayecto de la Línea A. El rezago de decisión comprendido entre la idea original y el inicio de las obras fue de 23 años. En Ciudad de México, unos estudiantes presentaron una propuesta en 1958, que finalmente fue concretada en 1967 cuando la ciudad contaba con cerca de 5 millones de habitantes para que, en 1969, se inauguraran dos tramos, el de Chapultepec y el de Zaragoza: el rezago fue de 9 años. En Caracas, la primera noticia data de 1947 y los decretos de expropiación de la tierra requerida para el primer tramo se expidieron a final de 1968 para que, en 1970, cuando la ciudad tenía 2,6 millones de habitantes, se inauguraran los trayectos Catia-Petare y Catia-El Silencio: el rezago fue de 21 ańos. En Lima se tuvo la idea hacia 1965 y las obras se iniciaron en 1986, para que en el 2011, cuando tenía 8,6 millones de habitantes, se inaugurara el primer tramo: el rezago fue de 21 ańos. La idea original en Río de Janeiro se remonta a 1950, habiéndose iniciado las obras en 1970 e inaugurado el primer tramo en Gloria en 1979: el rezago fue de 20 ańos. En Montreal, la idea original se remonta a 1902, pero se tiene la certeza de que se adoptó en 1910, no obstante que las obras se iniciaron a mediados de 1962, habiéndose inaugurado el primer trayecto hacia mediados de 1966 cuando la ciudad tenía 1,3 millones de habitantes: el rezago fue de 60 años.

El rezago de decisión es una noción útil que indica que cuando se difiere en demasía la ejecución de la inversión, el proyecto se torna menos viable desde cualquier punto de vista: el financiero, el ecológico, el político o el urbanístico, entre otros. Las cosas suben de 
precio y la incertidumbre macroeconómica sugiere aplazar la decisión, el deterioro del paisaje suele pesar más en la opinión que la potencial reducción de la emisión de gases tipo invernadero, la polarización política latente entre las élites nacionales y el potencial de algún alcalde capitalino de disputar en el futuro el comando centralista del país y, finalmente, los inconvenientes que generan las obras a los residentes que, aunque acostumbrados a sobrevivir en una ciudad en estado permanente de construcción / renovación, no dejan de cobrar políticamente por el fastidio que estas ocasionan.

¿Existe algún criterio que permita superar el rezago de decisión? La experiencia tokyoita puede ser de utilidad. Si hay algo en común con Tokyo son las elevadas densidades poblacionales alcanzadas en varias zonas de la ciudad, fenómeno que han aprovechado para garantizar el volumen más elevado de pasajeros para los sistemas metro y de ferrocarriles suburbanos (Sort, 2005, p. 96). La elevada densidad fue considerada explícitamente desde 1982 por el Conpes como una de las tres condiciones que justifican la inversión en un sistema metro. Otro aspecto en común es la escasa versatilidad de las vías urbanas para acoger gran cantidad de automóviles pues, de hecho, siendo uno de los países que más producen vehículos de uso particular, la tasa de motorización no es tan elevada como en otras metrópolis (p. 99). Un trazado que conecte las zonas más densas de la ciudad sería el más eficiente por cuanto garantizaría captar el flujo más elevado de pasajeros y solucionar el problema de movilidad al mayor contingente posible, demanda que además proveería los flujos de caja más regulares y elevados y, adicionalmente, elevaría la productividad laboral y académica de miles de residentes en la ciudad. Decisiones con base en la densidad permiten racionalizar la construcción de estaciones y con ello moderar el costo de la infraestructura de soporte y, simultáneamente, elevar la velocidad comercial del sistema. Esta experiencia no requiere de estudios costosos pues tanto la obra de Sort, como la gestión tokyoita de la movilidad cotidiana y las densidades poblacionales de Bogotá están al alcance de todos. La propuesta de JICA en 1996 ha sido la más coherente, y con ella se hubiese podido acotar el rezago de decisión.

\section{Rezago de financiación}

Las necesidades de financiación dependen de las de inversión. La financiación de la inversión de un sistema metro y de sus componentes tiene, como cualquier otra inversión productiva, un prerrequisito indeclinable: saber lo que se quiere. Cualquier suma puede lucir irrisoria o copiosa al desconocerse el diseño del proyecto, sus componentes y la naturaleza de los costos.

Hay una idea generalizada de que los sistemas de transporte masivo y, en particular, los sistemas metro solo pueden operar con un subsidio permanente; es decir, que son insostenibles financieramente con la mera tarifa. ¿Por qué ocurre esto? Por la naturaleza, magnitud y forma de imputación de los costos y, como resultado de esto, del cálculo de la tarifa técnica. No obstante, hay casos que contradicen esta idea. El Administrador Financiero de Transantiago paga a la Empresa 
Metro $\$ 349,25$ por cada pasajero transportado, cifra en pesos chilenos del 2016 que es el costo neto del servicio de transporte; es decir, la tarifa técnica. Por su parte, el usuario paga \$740; es decir, la tarifa al público. La diferencia se emplea para costear la operación de los buses por la vía de la integración tarifaria (El Mercurio, 2016).

$\mathrm{Al}$ encargar la estructuración financiera del sistema metro a un privado, a una banca de inversión, por ejemplo, no desaparece la responsabilidad política del alcalde, debido a que existen decisiones que en apariencia son de carácter técnico, pero que en la práctica tienen un contenido político ineludible. Una de ellas es precisamente la de la justificación de la tarifa al público y los efectos que esta decisión tiene en al menos tres aspectos inmanentes a la operación del sistema metro. El primero es el lapso de la depreciación de los activos que juega un papel decisivo en la financiación, así como los gastos de mantenimiento y de reposición, la obsolescencia y la posibilidad de que exista algún valor de salvamento de alguna parte del equipo. Los plazos de depreciación largos amortiguan las pérdidas, pero ellos solo pueden alcanzarse con costos de reposición y mantenimiento elevados que también impactan la tarifa técnica.

La segunda decisión -esencialmente política, aunque de apariencia técnica- es la tasa de retorno del proyecto que refrenda su factibilidad económica y que, en teoría, debe ser superior a la tasa de interés de los créditos contratados pues, cuando ocurre lo contrario, es previsible que en algún momento se incurra en la mora en el pago del servicio de la deuda $y$, por tanto, en mayores costos financieros.
Sobre este segundo aspecto no hay que perder de vista que lo que se descuenta es un flujo de caja que está determinado por la afluencia de pasajeros y, por tanto, por el trazado, siendo el más conveniente, como ya se argumentó, el que conecta las zonas más densamente pobladas de la ciudad.

El tercer factor que incide de manera decisiva en la tarifa técnica es la magnitud de los costos hundidos pues, entre mayor sea su proporción, menor será su impacto en la tarifa técnica, pero cuando una porción de ellos sale a flote es causal, normalmente, de la aparición de pérdidas contables que afectan la gestión del sistema. Los costos de los estudios y las consultorías de diseños pasados podrían considerarse dentro de este tipo de costos, así la solución propuesta no sea por la que se opte, pues el modelo público comercial, a diferencia de otras agencias de la administración pública, no opera con inversiones a fondo perdido.

Un aspecto final de no poca trascendencia: la posibilidad cierta de suspensión de obras por falta de recursos financieros. Este fenómeno ocurrió un año después de haberse iniciado las obras en Río de Janeiro -en 1971-, situación que se prolongó hasta 1974. La crisis económica y el terrorismo ocasionaron la suspensión de la construcción de la primera línea del metro de Lima que, no obstante, fue inaugurado en 1990 como una obra inservible, pudiéndose concluir para entrar efectivamente en funcionamiento regular en 2011. El reinicio de las obras es una tarea muy compleja pues, invariablemente, los costos se incrementan con el paso del tiempo. Paradójicamente, el costo político de la suspensión de las obras y de las externalidades pecuniarias 
que deben sufragar los contribuyentes es bajo pues, en la práctica, la promesa de reinicio y conclusión de las obras son asumidas como bandera de los programas de Gobierno de los candidatos perdedores y electos a las alcaldías. Esto es lo que ha ocurrido en los casos mencionados. Tal posibilidad se ha hecho explícita en los documentos de política del Gobierno nacional que, como en el caso del documento Conpes 2999, estableció que "el desarrollo de esta iniciativa no puede generar presión fiscal ni financiera sobre el Gobierno central"; es decir, que la estabilidad macroeconómica se impone ante cualquier tipo de contingencia.

\section{Rezago de instalación}

La irreversibilidad de las decisiones como el trazado, el número de estaciones y las tecnologías por emplear en la construcción del sistema metro de transporte colectivo de pasajeros (Alfonso, 2012a, pp. 329-335), hace que este tipo de inversiones afronte más riesgos que aquellas en las que existe movilidad espacial del capital y la incertidumbre tecnológica es menor. El margen de factibilidad económica se ha reducido paulatinamente al decir del decrecimiento de las tasas internas de retorno del sistema metro en sus diferentes versiones, lo que en buena medida se debe al cambio de parecer en cuanto a los trazados, a las fluctuaciones externas y a la indefinición de la estrategia financiera.

La apertura de la licitación para la construcción es una señal de que los rezagos de decisión y de financiación se han superado. Con justa razón, un inusitado optimismo acostumbra invadir el espíritu de los cuadros directivos de la administración local que, en no pocas ocasiones, llega a desbordarse al punto de hacerlos creer que la inauguración está a un paso, y que en las placas conmemorativas estarán sus nombres, algo invaluable para su proyecto político. Pero las contingencias aparecen desde antes de la apertura de la licitación y se prolongan hasta después de inaugurada la obra, siendo ellas de tres tipos: las de decisión, las de financiación y las derivadas de conductas antijurídicas. De las dos primeras se derivan responsabilidades directas de la acción u omisión del Estado cuyo daño debe ser reparado por la vía de la indemnización, mientras que la tercera deriva en prolongados litigios a no ser que haya una conciliación rápida que acota los costos judiciales.

Cuando el componente del trazado está claramente definido y se conoce el propósito principal del proyecto, cual es el de mejorar de manera sustancial las condiciones de movilidad cotidiana del mayor número posible de residentes, así como los secundarios que se engloban fundamentalmente en las mejoras en el urbanismo a lo largo y ancho del área de influencia inmediata de la línea, la compra de predios a los particulares debe anticiparse al anuncio del proyecto y no postergarse para después de haber sido anunciado. Si el Estado compra los predios antes del anuncio del proyecto, neutraliza la acción especulativa de los particulares y se sustrae de iniciar procesos de captura de plusvalía en los que se ha mostrado poco eficaz. Cuando la compra de predios no comienza con la antelación suficiente sobrevienen los procesos de expropiación por vía administrativa, generalmente con indemnización. La duración de los procesos es determinada, 
de una parte, por la capacidad de interferencia en el curso normal de los procesos de los profesionales litigantes que es considerable $y$, de la otra, por la eficacia de la justicia que está condicionada, en buena medida, por la congestión judicial. El hecho es que sin la disponibilidad de predios, la iniciación de las obras se puede retrasar originando el reclamo de indemnizaciones por parte del constructor. No deben pasarse por alto los imprevistos y otras contingencias: durante la construcción de la Línea Cuatro del sistema metro de São Paulo, en 2007, ocurrió un derrumbe que originó un socavón de 80 metros de diámetro, mientras que las lluvias del 2016 ocasionaron el derrumbe parcial del túnel de la Línea Dos en Santo Domingo.

Las contingencias de financiación afloran principalmente por los desajustes en el flujo de caja del proyecto durante las fases previas a la iniciación pero, con más regularidad, aparecen durante su ejecución. Además del riesgo de iliquidez surgido de la mencionada primacía de la estabilidad macroeconómica sobre los flujos financieros del proyecto en su etapa de construcción, existen otros riesgos asociados al carácter precio-aceptante de una economía pequeña y al riesgo cambiario. Puesto que el componente importado es elevado, la sensibilidad del proyecto a las fluctuaciones externas es considerable pues, de un lado, los precios relativos de los bienes de inversión se elevan por tratarse de precios cuasi-monopólicos y, en segundo lugar, el periodo del pacto revaluacionista fue el más propicio para la instalación del proyecto, a diferencia del actual en el que la devaluación galopante ha incidido poderosamente en el precio de los importables.

\section{REFLEXIONES FINALES}

La teoría de los rezagos distribuidos de la inversión productiva ha permitido analizar, de manera integral, los desaciertos acumulados durante 75 años por las políticas de transporte masivo en Bogotá en relación con la introducción del sistema metro. La procastinación administrativa en materia de movilidad ha ocasionado el aplazamiento de la decisión y, por ello, se perdió la oportunidad de orientar el crecimiento de la ciudad con esta infraestructura, como sí lo consiguieron aquellas metrópolis del continente que tomaron la decisión con celeridad. No obstante, ese discurso persiste en la actualidad cuando la estructura física de la ciudad presenta tal rigidez que difícilmente un sistema metro podrá reorientar su estructura económica y residencial para superar sus rasgos dominantes tales como la segregación socioespacial.

Durante ese lapso de tiempo, las decisiones cruciales de la política de movilidad se aplazaron produciendo un acumulado de déficits de movilidad, tanto en los modos como en los medios de transporte colectivo. La insostenibilidad del actual sistema integrado de transporte público es un acumulado histórico que se percibe de diferentes maneras: en lo económico se trata de un mercado con una elevada demanda en el que, sin embargo, pierden casi todos los agentes que participan; en lo ecológico, es un sistema entrópico (Alfonso, 
2015b) que dilapida una cantidad ingente de materia y de productividades laborales y académicas; y, en el plano político, es un contexto en el que los políticos acostumbran reeditar sus propuestas mesiánicas.

La congestión vehicular ha tenido varios determinantes, siendo el más difundido el de la escasez de vías. Los dirigentes gremiales acostumbran alinearse alrededor de este discurso para reclamar una ciudad dotada de autopistas urbanas en donde los vehículos particulares no tengan mayores impedimentos para su circulación. Esa alternativa es la más costosa para las metrópolis cuyos dirigentes incurrieron en rezagos de decisión virtualmente irresolubles en la actualidad. El ensanchamiento de las vías arterias conlleva la destrucción de stocks inmobiliarios con larga vida útil, al igual que la reubicación de las redes subterráneas de servicios públicos y colectivos, así como la construcción de tramos elevados o deprimidos en las intersecciones para que la vía expresa no sea interrumpida por una impertinente semaforización.

En tal contexto de una metrópoli construida y densamente poblada, el sistema metro tiene un objetivo central: resolver los problemas de movilidad cotidiana del mayor número posible de usuarios del transporte colectivo. Para alcanzar tal propósito, el trazado de la línea prioritaria intenta conectar el mayor número posible de las zonas con más densidad poblacional de las metrópolis. Esta es la regla con la que se han definido los trazados, las áreas de aferencia de pasajeros, el número de estaciones y la velocidad comercial (Alfonso, 2012a) en los sistemas más eficaces del mundo y, con ello, se ha superado rápidamente el rezago de decisión. Resuelto este rezago, los abordajes esperados son los máximos, quedando por resolver los montos de las tarifas técnicas y al público. Como ilustra el caso de Transantiago, la tarifa técnica puede ser inferior a la tarifa al público y, con ello, el rezago de financiación y los riesgos conexos se reducen pero, para ello, es necesario tomar decisiones sobre tres aspectos cruciales: la vida útil del proyecto y las inversiones en mantenimiento y reposición, los costos hundidos y la rentabilidad interna del proyecto. Estas decisiones las puede sugerir cualquier banca de inversión pero, finalmente, se tomarán en la esfera política. Superados los rezagos de decisión y de financiación, la instalación puede ser más expedita pero no exenta de riesgos como la disponibilidad de suelo para las obras civiles y otros derivados de la sismorresistencia en las zonas de trazado.

La política de transporte masivo de pasajeros es pública, no obstante que se opte por un modelo de gestión comercial. La responsabilidad del Estado no se diluye en las bancas de inversión que sugieren decisiones o en las sociedades de capital que persiguen la eficiencia en la asignación de recursos con independencia de las decisiones políticas. Un sistema eficaz de transporte masivo de pasajeros cuya prioridad sea la de conectar las zonas más densas de la ciudad privilegia la opción del sistema metro por varias razones, entre las que la principal es que goza de la frecuencia con que no cuenta Transmilenio, y otras, como el incremento de la velocidad comercial, refuerzan esta decisión, a no ser que se conciba al metro como una de sus rutas alimentadoras. 
La sostenibilidad del sistema de transporte masivo de Bogotá requiere de políticas osadas que promuevan la resolución del déficit de capital de movilidad que comprende no solo la infraestructura vial y el parque de movilidad, sino también su aparato de dirigencia y de gestión, cuyo diseño aún se encuentra en ciernes pues, en efecto, no se ha aprendido de los errores ni de los escasos aciertos, así como tampoco se conocen las inflexibilidades infraestructurales de una ciudad construida que en 2017 alcanzará los ocho millones de habitantes, en 2027 los nueve y los diez en 2041, momentos en los que se prevé que se reitere la pregunta ¿y por qué razones Bogotá no cuenta con al menos una línea metro? Tal osadía debe concretarse en eliminar los factores que han motivado los tres tipos de rezagos y que, en la coyuntura reciente, se han exacerbado: cambio de diseños que prolongan el rezago de decisión, uso inadecuado de la vigencias futuras que alientan el rezago de financiación y, en consecuencia, se difiere nuevamente el inicio de las obras con lo que el rezago de instalación continúa en aumento.

\section{REFERENCIAS}

Alcaldía Mayor de Bogotá, Bogotá Humana, Metro de Bogotá (s. f.). Evaluación socioeconómica ex ante del proyecto "Primera Linea del Metro de Bogotá”. Recuperado de http://webidu.idu.gov. co:9090/jspui/bitstream/123456789/112844/1/ ME008347.pdf

Alfonso, R. Ó. y Amézquita, L. (2016). Housing market segmentation and real estate bubble threat, Bogota
2006-2015. Ponencia presentada en la 23rd Annual Conference of European Real Estate Society, University of Regenesburg, Alemania. Alfonso, R. Ó. (2015a). ¡La vivienda que quiero está muy cara! Un análisis sobre la segmentación del mercado residencial, la alarma de burbuja inmobiliaria en la zona metropolitana de Bogotá y la ineficiencia asignativa del mercado del suelo. En De los Andes al litoral. Estudios sobre vivienda y suelo en Colombia. Bogotá: Universidad de la Salle.

Alfonso, R. Ó. (2015b). Segmentación del espacio residencial y colapso de la movilidad cotidiana en la zona metropolitana de Bogotá. En Continuidades, rupturas y emergencias: trayectorias de las desigualdades en las ciudades de América Latina. México D. F.: Centro de Investigaciones sobre América Latina y el Caribe, Programa Universitario de Estudios de la Ciudad, Posgrado en Estudios Latinoamericanos, Universidad Nacional Autónoma de México.

Alfonso, R. Ó. (2012a). Bogotá segmentada: reconstrucción histórico-social de la estructuración residencial de una metrópoli latinoamericana. En Colección Economía Institucional Urbana. Bogotá: Universidad Externado de Colombia.

Alfonso, R. Ó. (2012b). Urbanismo ordenado pero pasteurizado: origen del corporatismo y orden socio-espacial en Bogotá hacia 1910. En El centro tradicional de Bogotá, valor de uso popular y patrimonio arquitectónico de la ciudad. Colección Economía Institucional Urbana. Bogotá: Universidad Externado de Colombia.

Alonso-Neira, M., Galledo-Lozada, R. y Pires-Jiménez L. (2013). La ampliación del Metro en la periferia de Madrid (1999-2011). Revista EURE, 39, (118). Santiago de Chile: puc. 
Bour, E. (2004). Teoría de la inversión. Recuperado de http://www.ebour.com.ar/pdfs/Teoria \%20 de \%20la \%20inversion.pdf.

Clark, G. y Moir, E. (2015). Density: drivers, dividends and debates. London: Urban Land Institute.

Concejo de Bogotá (2016). Acuerdo 645 de 2016: por el cual se adopta el Plan de desarrollo económico, social, ambiental y de obras públicas para Bogotá D.C. 2016-2020 "Bogotá mejor para todos". Bogotá: Concejo Distrital.

Conpes (2010). Documento 3677: Movilidad integral para la Región Capital Bogotá - Cundinamarca. Bogotá: Departamento Nacional de Planeación.

Conpes (2000). Documento 3093: Sistema de servicio urbano de transporte masivo de pasajeros de Bogota -Seguimiento-. Bogotá: Departamento Nacional de Planeación.

Conpes (1998). Documento 2999: Primera Linea Metro para Bogotá. Bogotá: Departamento Nacional de Planeación.

Conpes (1982). Documento DNP-1.885-UINF: Proyecto Metro Bogotá. Bogotá: Departamento Nacional de Planeación.

Cuervo, L. M. (1994). Crisis y regulación de los servicios colectivos y domiciliarios en Colombia. Cuadernos de Economía, XIV (20), 295-312.

De Grange, C. L. (2010). El gran impacto del Metro. En Revista EURE, 36 (107). Santiago de Chile, puc. DNP (s. f.). DNP advierte que se avecina colapso de movilidad en las principales capitales. Recuperado de https://www.dnp.gov.co/Paginas/DNP \%20 advierte \%20que \%20se \%20avecina \%20colapso $\% 20$ de $\% 20$ movilidad \%20en \%20las \%20 principales $\% 20$ capitales.aspx

Duarte, V. C. (2015). Movilidad urbana. ¿Cómo definir un sistema de transporte público colectivo (sitp), en Bogotá D.C, Colombia? Cuadernos
Latinoamericanos de Administración, XI (20). Bogotá: Universidad El Bosque.

Ebour, E. (2004). Teoría de la Inversión. Recuperado de http://www.ebour.com.ar/pdfs/Teoria \%20 de \%20la \%20inversion.pdf

Eisner, R. y Nadiri, M. I. (1968). Investment behavoir and neo-classical theory. The Review of Economics and Statistics. Recuperado de http://www.econ. nyu.edu/user/nadiri/pub10.pdf (20/5/2016).

Escobar, A. (2016). “Se espera que el primer tren esté rodando en el ańo 2020". Revista Semana, edición digital del 22/04/2106.

Flyvberg, B., Garbuio, M. y Lovallo, D. (2009). Delusion and Deception in Large Infraestructure Projects: Two Models for Explaining and Preventing Executiv Disaster. California Management Review, 51 (2). Recuperado de file://C:/Users/ Propietario/Downloads/ssRn-id2229781.pdf

El Mercurio, Chile (2016). Costo real por cada pasajero que se transporta en el Metro de Santiago llega a \$350. Recuperado de http://www.emol.com/ noticias/Nacional/2016/02/17/788688/Costoreal-por-cada-pasajero-que-se-transporta-en-elMetro-de-Santiago-llega-a-350.html

Jaramillo, S. (2009). Hacia una teoría de la renta del suelo urbano. Bogotá: CEDE - Facultad de Economía de la Universidad de los Andes.

Jorgenson, D. (1967). The theory of investment behavoir. En Determinants of investment behavior. National Bureau of Economic Research. Recuperado de http://www.nber.org/chapters/ c1235.pdf

Guitton, H. y Koyck, L. M. (1955). Distributed Lags and Investment Analysis. Revue Économique, 6 (1). Recuperado de http://www.persee.fr/doc/ reco_0035-2764_1955_num_6_1_407098_ t1_0127_0000_001 
Mauttone, A. (2005). Optimización de recorridos y frecuencias en sistemas de transporte urbano colectivo [Tesis de Maestría en Informática]. Montevideo: Facultad de Ingeniería - Universidad de La República. Recuperado de http://www.fing.edu. uy/inco/pedeciba/bibliote/tesis/tesis-mauttone. $\operatorname{pdf}(5 / 9 / 2016)$.

Miller, R. (1967). On Jorgenson. En Determinant of Investment Behavior. National Bureau of Economic Research. Recuperado de http://www. nber.org/chapters/c1235.pdf.

Mohan, D. (2008). Mythologies, Metros \& Future Urban Transport. TRIPP Report Series. Delhi: Indian Institute of Technology. Recuperado de http://tripp. iitd.ernet.in/delhibrts/metro/Metro/Metro \%20 Mythology08.pdf

Oliveira, B. y Texeira, J. (1999). Impacto de la inversión pública sobre la inversión privada en Brasil: 1947-1990. Revista de la Cepal (67). Santiago de Chile: Cepal. Recuperado de http://archivo. cepal.org/pdfs/revistaCepal/Sp/067071080.pdf (5/9/2016).

Rodríguez, A. y Abramo, P. (2011). Grandes proyectos urbanos y su impacto en el mercado del suelo urbano. Cambridge, MA: Lincoln Institute of Land Policy. Recuperado de http://www.ceur-conicet. gov.ar/imagenes/Rodriguez_Abramo_Grandes_ proyectos_urbanos_y_su_impacto_en_el_mercado_de_suelo_urbano \%5B1 \%5D.pdf

Rodríguez, D. y Vergel, E. (2014). Desarrollo urbano orientado a los sistemas de transporte público masivo tipo BRT (Bus Rapid Transit) en Quito y Bogotá. Recuperado de https://www.lincolninst. edu/pubs/2456_Desarrollo-urbano-orientadoa-los-sistemas-de-transporte-p \%C3 \%BAblicomasivo-tipo-BRT--Bus-Rapid-Transit--en-Quitoy-Bogot $\%$ C3 \%A1
Secretaría Distrital de Movilidad (2014). Movilidad en cifras 2014. Bogotá: Distrito Capital, Alcaldía Mayor.

Secretaría Distrital de Planeación (2008). Principales estudios de metro para la Ciudad de Bogotá en los últimos 30 años. Sintesis de Coyuntura (58). Bogotá: SDP.

Sort, J. J. (2005). Redes metropolitanas. Barcelona: Editorial Gustavo Gili.

Suárez, A. (2015). ¿¿Por qué más de 70 años sin metro en Bogotá? El Espectador.

Thomson, I. (1997). ¿Por qué las inversiones en transporte público no reducen la congestión de tránsito urbano? Revista de la CEPAL (61). Recuperado de http://repositorio.cepal.org/bitstream/handle/11362/12049/061107118_es.pdf?sequence=1 $(18 / 5 / 2016)$.

Tobin, J. (1969). A general equilibrium approach to monetary theory. En Journal of Money, Credit and Bank. Recuperado de http://www. jstor.org/stable/1991374? origin=JSTORpdf\&seq=1\#page_scan_tab_contents

Universidad Nacional de Colombia (2010). Informe final validación de los estudios del Metro en Bogotá: correspondiente al informe 4-B del contrato UN-DNP 409. Bogotá: Universidad Nacional de Colombia. Recuperado de http://www.institutodeestudiosurbanos.info/dmdocuments/cendocieu/ coleccion_digital/Estudios_Metro/Informe_Final_Validacion-un-2010.pdf) (28/4/2016).

Weisbrod, G. (2009). Economic Impact of Public Transportation Investment. Boston, American Public Transportation Association. Recuperado de http:// www.apta.com/resources/reportsandpublications/Documents/economic_impact_of_public_transportation_investment.pdf(5/9/2016). Wingo, L. (1972). Transporte y suelo urbano. Barcelona: Oikos. 


\section{Páginas web visitadas}

Nace Empresa Metro de Bogotá, S. A. http://app.idu. gov.co/seccion_metro_ASP/

Subte, Metro de Buenos Aires. http://www.buenosaires. gob.ar/subte

Metrôrio, Metro de Rio de Janeiro. https://www.metrorio.com.br/

Metro de Ciudad de México. http://www.metro.cdmx. gob.mx/

Línea 1, Metro de Lima. http://www.lineauno.pe/

STM, Metro de Montreal. http://www.stm.info/en/info/ networks/metro 